\title{
A Generalisation of Pre-logical Predicates to Simply Typed Formal Systems
}

\author{
Shin-ya Katsumata \\ Laboratory for Foundations of Computer Science \\ School of Informatics, The University of Edinburgh, \\ King's Buildings, Edinburgh EH9 3JZ, UK \\ E-mail address: S. Katsumata@sms.ed.ac.uk
}

\begin{abstract}
We generalise the notion of pre-logical predicates [HSO2] to arbitrary simply typed formal systems and their categorical models. We establish the basic lemma of pre-logical predicates and composability of binary pre-logical relations in this generalised setting. This generalisation takes place in a categorical framework for typed higher-order abstract syntax and semantics [Fio02,MS03].
\end{abstract}

\section{Introduction}

Pre-logical predicates (relations) [HSO2] are a generalisation of logical predicates. They are defined for the simply typed lambda calculus and its set-theoretic environmental models called lambda applicative structures [Mit96]. Two important properties are enjoyed by pre-logical predicates but not logical predicates. One is that pre-logical predicates are equivalent to predicates satisfying the basic lemma (interpretation of all terms respects predicates - this is the key to many applications of logical relations), and the other is that binary pre-logical relations are closed under relational composition.

We aim to generalise pre-logical predicates from the simply typed lambda calculus to arbitrary simply typed formal systems (we just say typed formal system below) and their categorical models, then show that the above important properties hold in this generalised setting.

This generalisation enables us to extend pre-logical predicates systematically to other calculi, such as lambda calculus with various type constructors and variable binders, and calculi other than lambda calculus, such as logics and process calculi. This opens up the possibility of characterising observational equivalence [HSO2] and constructive data refinement [HLST00] in various non-lambda calculi.

There are three underlying elements on which pre-logical predicates are defined: syntax (normally the simply typed lambda calculus), semantics (set-theoretic environmental models) and predicates (as subsets of carrier sets). We generalise these three elements along the following dimensions:

- We generalise syntax to an arbitrary typed formal system described by a typed binding signature [MS03]. A typed formal system is a formal system whose inference rules fit within the following scheme:

$$
\frac{\Gamma, \Gamma_{1} \vdash M_{1}: \tau_{1} \quad \cdots \quad \Gamma, \Gamma_{m} \vdash M_{m}: \tau_{m}}{\Gamma \vdash o\left(\Gamma_{1} \cdot M_{1}, \cdots, \Gamma_{m} \cdot M_{m}\right): \tau}
$$


This is general enough to subsume various simple type systems and calculi such as the simply typed lambda calculus, many-sorted first-order logic, pi-calculus, etc.

- We generalise from set-theoretic to category-theoretic semantics. Following the principle of categorical semantics, we give a semantics of a typed formal system in a Cartesian category $\mathbb{C}$ by mapping types to objects and terms to morphisms in $\mathbb{C}$.

- As we move to category theory, we need to change the notion of predicates from subsets to appropriate category-theoretic constructs. We use subscones, which is a mild generalisation of the injective scones of [MS93].

We represent all three elements as objects and morphisms in the category of presentation models $\mathbf{M}_{T}$, where $T$ is the set of types [MS03]. In this category, the collection of well-formed terms modulo $\alpha$-equivalence is represented as the initial algebra of the endofunctor corresponding to a typed binding signature.

After this generalisation, we formulate pre-logical predicates and predicates satisfying the basic lemma, and show their equivalence. Then we show the composability of binary pre-logical relations.

We look at three examples of pre-logical predicates, i) the relationship between pre-logical predicates for combinatory algebra and those for lambda calculus, ii) the connection between pre-logical predicates and lax logical predicates [PPST00] and iii) a characterisation of elementary submodels of first-order classical logic by a pre-logical relation.

Structure of This Paper The generalisation of pre-logical predicates takes place in the following way. In section 2, we first introduce a category of presentation models $\mathbf{M}_{T}$, and typed binding signatures as a description of typed formal systems. We give a categorical semantics of typed formal systems in a very weak sense. We introduce a formulation of predicates with respect to this semantics, using subscones. All three elements (syntax, semantics and predicates) are expressed in category $\mathbf{M}_{T}$. Then we formulate pre-logical predicates and predicates satisfying the basic lemma in section 3 . The basic idea of the formulation of pre-logical predicates is that the inverse image of a predicate along the meaning function has an algebra structure. We show that predicates satisfying the basic lemma and pre-logical predicates are equivalent. Composition of binary pre-logical relations is discussed in section 4 . In section 5 , we look at three examples of pre-logical predicates. Proofs are attached in the appendix.

Related Work First, we briefly consider formulations of logical predicates. Logical predicates (and relations) have been widely used as a tool to study properties of the simply typed lambda calculus. Ma and Reynolds [MR92] formulated logical predicates as Cartesian closed functors from the free CCC $\mathbf{L}$ to $\operatorname{Pred}(G)$. Hermida [Her93] pointed out that $\operatorname{Pred}(G)$ can be replaced with the total category $\mathbb{E}$ of a fibration $p: \mathbb{E} \rightarrow \mathbb{B}$, provided that CCC structures on $\mathbb{B}$ can be lifted to $\mathbb{E}$. Plotkin et al. [PPST00] introduced a weakening of Ma and Reynolds' formulation called Lax logical predicates, which are functors from $\mathbf{L}$ to $\operatorname{Pred}(G)$ preserving only finite products. The basic lemma still holds for lax logical predicates, and furthermore the converse holds. In this sense lax logical predicates and pre-logical predicates are the same. They extended lax logical 
predicates from the lambda calculus to the language described by a finitary monad over Cat extending finite product structure.Lax logical predicates are also extended to the computational lambda calculus [KP99]. Binary lax logical relations are closed under composition.

Kinoshita, et al. [KOPT97] proposed a generalisation of logical relations called $L$ relations. Their framework is also parameterised by a finitary monad $L$ over Cat, which allows us to generalise the language from the lambda calculus. They used category objects in Cat to formulate the composition of L-relations.

Leiß[Lei01] extended pre-logical predicates to system $F \omega$, and characterised observational equivalence in terms of existence of binary pre-logical relation.

An application of binary pre-logical relations is to characterise observational equivalence between two models of a language [Lei01,HS02,Kat03].

This work refers to the framework by Fiore [Fio02] and Miculan and Scagnetto [MS03] on a categorical model of typed higher-order abstract syntax. This framework is a natural extension of the one considered in [FPT99,Hof99] to take types into account.

Convention We identify a set and its discrete category. We assume that all categories appeared in this paper are locally small. By a Cartesian category we mean a category with chosen finite products. We fix a countably infinite set of variables $X$ (ranged over by $x, y, z)$. For a finite set $A$, by $|A|$ we mean the number of elements in $A$. We use $\vec{A}$ for a sequence of meta variables, like $A_{1}, \cdots, A_{n}$.

\section{Preliminaries}

Category of Presentation Models We introduce the category of presentation models [MS03] plus some auxiliary categories for the following sections. We represent all three elements involved in the notion of pre-logical predicates (syntax, semantics and predicates) in this category.

Let $T$ be the set of types, whose elements are ranged over by $\tau, \sigma$. A context (ranged over by $\Gamma$ ) is a function from a finite subset of $X$ to $T$. A context renaming from $\Gamma$ to $\Gamma^{\prime}$ is a function $f: \operatorname{dom}(\Gamma) \rightarrow \operatorname{dom}\left(\Gamma^{\prime}\right)$ such that $\Gamma^{\prime} \circ f=\Gamma$. They form the category of contexts $\mathbf{C}_{T}{ }^{1}$ with the initial object given by the empty context ! : $\emptyset \rightarrow T$ and binary coproducts given by cotupling of contexts $\left[\Gamma, \Gamma^{\prime}\right]: \operatorname{dom}(\Gamma)+\operatorname{dom}\left(\Gamma^{\prime}\right) \rightarrow T$. By $\Gamma, \Gamma^{\prime}$ we mean the coproduct of contexts $\Gamma$ and $\Gamma^{\prime}$ whose domains are disjoint. We fix a variable $x \in X$ and define $\langle-\rangle: T \rightarrow \mathbf{C}_{T}$ by $\langle\tau\rangle=\{x \mapsto \tau\}$. We assume that each variable $x \in \operatorname{dom}(\Gamma)$ has an index number denoted by $\gamma(x) \in\{1, \cdots,|\operatorname{dom}(\Gamma)|\}$.

We define the ambient category $\mathbf{S}_{T}=$ Set $^{\mathbf{C}_{T}}$. Category $\mathbf{S}_{T}$ has small limits and colimits, and has a context extension operator $\left(\delta_{\tau} A\right)(\Gamma)=A(\Gamma+\langle\tau\rangle)$. In fact $\delta_{\tau} A$ is isomorphic to $V_{\tau} \Rightarrow A$, where $V_{\tau}(\Gamma)$ is the presheaf of variables of type $\tau$, defined to be $\mathbf{C}_{T}(\langle\tau\rangle, \Gamma) \cong\{x \mid \Gamma(x)=\tau\}$, thus $\delta_{\tau}$ has a left adjoint. Moreover it has a right

\footnotetext{
${ }^{1}$ Category $\mathbf{C}_{T}$ can be described as the comma category $I \downarrow T$ where $I: \mathbf{X} \hookrightarrow$ Set is the inclusion functor of the full subcategory $\mathbf{X}$ of Set whose objects are finite subsets of $X$ [Fio02,MS03]. It is a free co-Cartesian category generated from $T$.
} 
adjoint ([MS03], proposition 2), thus preserves both limits and colimits. We write $\delta_{\vec{\tau}}$ for the composition $\delta_{\tau_{1}} \circ \cdots \circ \delta_{\tau_{n}}$.

The category of presentation models $\mathbf{M}_{T}$ is defined to be $\left(\mathbf{S}_{T}\right)^{T} \cong \mathbf{S e t}^{\mathbf{C}_{T} \times T}$. It also has small limits and colimits.

Syntax: Typed Binding Signature A typed binding signature (ranged over by $\Pi$ ) is a tuple $(T, O)$ where $T$ is the set of types (ranged over by $\tau, \sigma$ ) and $O$ is the set of operators (ranged over by $o$ ), each of which is a pair of an operator symbol $s$ and its arity $\left(\left(\overrightarrow{\tau_{1}}, \sigma_{1}\right), \cdots,\left(\overrightarrow{\tau_{m}}, \sigma_{m}\right), \tau\right) \in\left(T^{+}\right)^{*} \times T$. We write $s^{\left(\overrightarrow{\tau_{1}}, \sigma_{1}\right), \cdots,\left(\overrightarrow{\tau_{m}}, \sigma_{m}\right) \rightarrow \tau}$ for such a pair in $O^{2}$, and $o^{\tau} \in O$ for an operator whose result type is $\tau$. A typed firstorder signature (ranged over by $\Sigma$ ) is just a typed binding signature $(T, O)$ such that for all $s^{\left(\overrightarrow{\tau_{1}}, \sigma_{1}\right), \cdots,\left(\overrightarrow{\tau_{m}}, \sigma_{m}\right) \rightarrow \tau} \in O, \overrightarrow{\tau_{i}}=\epsilon$. It coincides with the notion of many-sorted signature.

A typed binding signature $\Pi$ specifies a typed formal system. We first define raw- $\Pi$ term (ranged over by $M, N)$ by the BNF $M::=x^{\tau} \mid o\left(\overrightarrow{x^{\tau}} \cdot M, \cdots, \overrightarrow{x^{\tau}} \cdot M\right)$. In this BNF, $\overrightarrow{x^{\tau}} \cdot M$ means binding of variables $\overrightarrow{x^{\tau}}$ in $M$. As usual, we identify $\alpha$-equivalent terms. The typed formal system is a system to derive judgment $\Gamma \vdash_{\Pi} M: \tau$, where $\Gamma$ is an object in $\mathbf{C}_{T}$. The system consists of the following rules for variables and operators.

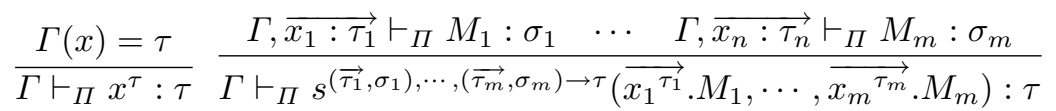

Example 1. 1. Let $B$ be a set. By Typ $\Rightarrow(B)$ we mean the set defined by the BNF $\tau::=b \mid \tau \Rightarrow \tau$ where $b \in B$. The typed binding signature $\Pi_{\lambda}$ for the simply typed lambda calculus is defined to be $\left(\mathbf{T y p} \Rightarrow(B),\left\{\operatorname{lam}^{\left(\tau, \tau^{\prime}\right) \rightarrow \tau \Rightarrow \tau^{\prime}}, \operatorname{app}^{\tau \Rightarrow \tau^{\prime}, \tau \rightarrow \tau^{\prime}}\right\}\right)$ where $\tau, \tau^{\prime}$ ranges over $\mathbf{T y} \mathbf{p} \Rightarrow(B)$.

2. The typed first-order signature for combinatory logic is

$$
\Sigma_{C L}=\left(\mathbf{T y p}^{\Rightarrow}(B),\left\{\operatorname{app}^{\tau \Rightarrow \tau^{\prime}, \tau \rightarrow \tau^{\prime}}, \mathrm{S}^{\left(\tau \Rightarrow \tau^{\prime} \Rightarrow \tau^{\prime \prime}\right) \Rightarrow\left(\tau \Rightarrow \tau^{\prime}\right) \Rightarrow \tau \Rightarrow \tau^{\prime \prime}}, \mathbf{K}^{\tau \Rightarrow \tau^{\prime} \Rightarrow \tau}\right\}\right)
$$

where $\tau, \tau^{\prime}, \tau^{\prime \prime}$ ranges over $\mathbf{T y} \mathbf{p}^{\Rightarrow}(B)$.

3. Let $\Sigma=\left(T_{0}, O_{0}\right)$ be a typed first-order signature. The typed binding signature for first-order classical logic over $\Sigma$ is

$$
\Pi_{\Sigma \text {-fol }}=\left(T_{0} \cup\{\boldsymbol{\Omega}\}, O_{0} \cup\left\{\text { exists }^{(\tau, \boldsymbol{\Omega}) \rightarrow \boldsymbol{\Omega}} \mid \tau \in T_{0}\right\} \cup\left\{\text { not }^{\boldsymbol{\Omega} \rightarrow \boldsymbol{\Omega}}, \text { or }^{\boldsymbol{\Omega}, \boldsymbol{\Omega} \rightarrow \boldsymbol{\Omega}}\right\}\right)
$$

The typed formal system described by $\Pi$ determines an object $S_{\Pi}(\tau)(\Gamma)=\left\{M \mid \Gamma \vdash_{\Pi}\right.$ $M: \tau\}$ in $\mathbf{M}_{T}$. This object can be characterised as an initial algebra of the functor associated to $\Pi$ by $(\Pi A) \tau=V_{\tau}+\coprod_{s\left(\overrightarrow{\tau_{1}}, \sigma_{1}\right), \cdots,\left(\overrightarrow{\tau_{m}}, \sigma_{m}\right) \rightarrow \tau \in O}\left(\prod_{i=1}^{m} \delta_{\overrightarrow{\tau_{i}}}\left(A \sigma_{i}\right)\right)$ together with the $\Pi$-algebra structure $\iota_{\Pi}: \Pi S_{\Pi} \rightarrow S_{\Pi}$ corresponding to the inference rules ([MS03], theorem 1).

\footnotetext{
${ }^{2}$ This definition of typed binding signature is a special case of the one in [MS03] where the set of types allowed for variables is equal to the set of all types.
} 
Semantics: Very Weak Categorical Model We formulate a semantics of a typed formal system $\Pi=(T, O)$ by a morphism to the object in $\mathbf{M}_{T}$ which reflects a Cartesian category. The notion of semantics considered here is very weak in the sense that it does not exploit any categorical structure other than finite products. The semantics keeps the basic principle of categorical model theory: that is, types are interpreted as objects and terms are interpreted as morphisms.

An interpretation of types is just a functor $F: T \rightarrow \mathbb{C}$ where $\mathbb{C}$ is a Cartesian category. We extend it to the functor $F^{*}: \mathbf{C}_{T} \rightarrow \mathbb{C}^{o p}$ by $F^{*} \Gamma=\prod_{i=1}^{|\operatorname{dom}(\Gamma)|} F\left(\Gamma\left(\gamma^{-1}(i)\right)\right)$, which preserves finite products in $\left(\mathbf{C}_{T}\right)^{o p}$. We write $s_{\Gamma, \Gamma^{\prime}}: F^{*} \Gamma \times F^{*} \Gamma^{\prime} \rightarrow F^{*}(\Gamma+$ $\left.\Gamma^{\prime}\right)$ for the natural isomorphism. For an interpretation of types $F: T \rightarrow \mathbb{C}$, we define the clone of typed operations $H^{F}$ by $H^{F}(\tau)(\Gamma)=\mathbb{C}\left(F^{*} \Gamma, F \tau\right)$. Let $\mathbb{D}$ be a Cartesian category. For a functor $G: \mathbb{C} \rightarrow \mathbb{D}$ preserving finite products strictly, we define a morphism $H^{G}: H^{F} \rightarrow H^{G F}$ in $\mathbf{M}_{T}$ by $H^{G}(\tau)(\Gamma)\left(f: F^{*} \Gamma \rightarrow\right.$ $F \tau)=G f:(G F)^{*} \Gamma=G F^{*} \Gamma \rightarrow G F \tau$. A categorical interpretation of $\Pi$ consists of a Cartesian category $\mathbb{C}$, an interpretation of types $F: T \rightarrow \mathbb{C}$ and a morphism $m: S_{\Pi} \rightarrow H^{F}$ in $\mathbf{M}_{T}$ called interpretation of terms, which assigns to a well-formed term $\Gamma \vdash_{\Pi} M: \tau$ a morphism $m(M): F^{*} \Gamma \rightarrow F \tau$. We use the notation $\mathbb{C} \llbracket-\rrbracket_{F}$ to represent a categorical interpretation. We define the product of categorical interpretations $\left(\mathbb{C}, F_{1}, m_{1}\right),\left(\mathbb{D}, F_{2}, m_{2}\right)$ to be $\left(\mathbb{C} \times \mathbb{D},\left\langle F_{1}, F_{2}\right\rangle,\left(m_{1}, m_{2}\right)\right)$ where $\left(m_{1}, m_{2}\right)$ is defined by $\left(m_{1}, m_{2}\right)(\tau)(\Gamma)=\left(m_{1}(\tau)(\Gamma), m_{2}(\tau)(\Gamma)\right)$.

Often, $H^{F}$ is equipped with a $\Pi$-algebra structure. In this case we can obtain an interpretation of terms by the initiality of $S_{\Pi}$. This is the initial algebra semantics for typed binding signature ([FPT99,MS03]). ${ }^{3}$

To specify a $\Pi$-algebra structure over $H^{F}$, it is sufficient to specify a morphism $u_{o}$ : $\prod_{i=1}^{m} \delta_{\overrightarrow{\tau_{i}}}\left(H^{F} \sigma_{i}\right) \rightarrow H^{F} \tau$ in $\mathbf{S}_{T}$ for each operator $o \in O$ of arity $\left(\overrightarrow{\tau_{1}}, \sigma_{1}\right), \cdots,\left(\overrightarrow{\tau_{m}}, \sigma_{m}\right) \rightarrow$ $\tau$. Together with the mapping $v^{\tau}: V_{\tau} \rightarrow H^{F} \tau$ defined to be $v^{\tau}(\Gamma)(x)=\pi_{\gamma(x)}$, we obtain a $\Pi$-algebra structure over $H^{F}$ by $\left[v^{-}, u_{o^{-}}\right]_{o^{-} \in O}:\left(\Pi H^{F}\right) \rightarrow H^{F}$.

Example 2. (Continued from example 1)

1. Let $F_{\lambda}: \mathbf{T y p} \Rightarrow(B) \rightarrow \mathbb{C}$ be an interpretation of types satisfying $F_{\lambda}\left(\tau \Rightarrow \tau^{\prime}\right)=$ $F_{\lambda}(\tau) \Rightarrow F_{\lambda}\left(\tau^{\prime}\right)$. The morphisms in $\mathbf{S}_{T}$ given by $\left(u_{\operatorname{lam}\left(\tau, \tau^{\prime}\right) \rightarrow \tau \Rightarrow \tau^{\prime}}\right)_{\Gamma}(f)=\lambda(f \circ$

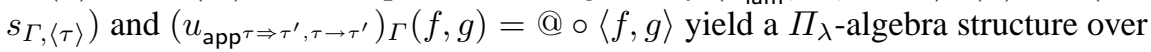
$H^{F_{\lambda}}$. The initial algebra semantics coincides with the standard semantics of the simply typed lambda calculus in $\mathbb{C}$.

2. Let $\Sigma=\left(T_{0}, O_{0}\right)$ be a typed first-order signature. A many-sorted $\Sigma$-algebra $\mathcal{A}$ consists of a $T_{0}$-indexed family of sets $A: T_{0} \rightarrow$ Set called carrier sets and an assignment of a function $o_{\mathcal{A}}: A^{\tau_{1}} \times \cdots \times A^{\tau_{n}} \rightarrow A^{\tau}$ to each operator $o \in O_{0}$ of arity $\tau_{1}, \cdots, \tau_{n} \rightarrow \tau$.

To each operator $o \in O_{0}$, we assign a morphism $\left(u_{o}\right)_{\Gamma}\left(f_{1}, \cdots, f_{n}\right)=o_{\mathcal{A}} \circ$ $\left\langle f_{1}, \cdots, f_{n}\right\rangle$ in $\mathbf{S}_{T}$. This yields a $\Sigma$-algebra structure over $H^{A}$ and the interpretation of terms, namely $\mathcal{A} \llbracket-\rrbracket: S_{\Sigma} \rightarrow H^{A}$.

\footnotetext{
${ }^{3}$ We note that interpretations of terms are not restricted to algebra morphisms. The reason is to cover the interpretation of terms which is obtained by composition of morphisms of different algebras. This case is considered in example 5 .
} 
3. Let $\Sigma=\left(T_{0}, O_{0}\right)$ be a typed first-order signature and $\mathcal{A}$ be a many-sorted $\Sigma$ algebra. We give a categorical semantics of $\Pi_{\Sigma \text {-fol }}$ in Set, which coincides with the standard interpretation of the first-order classical logic in the model constructed over $\mathcal{A}$. The interpretation of types $I_{\mathcal{A}}: T_{0} \cup\{\boldsymbol{\Omega}\} \rightarrow$ Set is given by $I_{\mathcal{A}}(\boldsymbol{\Omega})=$ $\mathbf{2}=\{\top, \perp\}$ and $I_{\mathcal{A}}(\tau)=A^{\tau}$ for all $\tau \in T_{0}$. To give an interpretation of terms, we specify the following morphisms for each operator.

$$
\begin{aligned}
& \left(u_{s^{\tau_{1}}, \cdots, \tau_{n} \rightarrow \tau}\right)_{\Gamma}\left(f_{1}, \cdots, f_{n}\right)=s_{\mathcal{A}} \circ\left\langle f_{1}, \cdots, f_{n}\right\rangle \quad\left(s^{\tau_{1}, \cdots, \tau_{n} \rightarrow \tau} \in O_{0}\right) \\
& \left(u_{\text {exists }(\tau, \Omega) \rightarrow \Omega}\right)_{\Gamma}(f)(\rho)=\top \Longleftrightarrow \exists x \in A^{\tau} . f(\rho, x)=\top \\
& \left(u_{\text {not } \Omega \rightarrow \Omega}\right)_{\Gamma}(f)(\rho)=\top \Longleftrightarrow f(\rho)=\perp \\
& \left(u_{\text {or } \Omega, \Omega \rightarrow \Omega}\right)_{\Gamma}\left(f_{1}, f_{2}\right)(\rho)=\top \Longleftrightarrow f_{1}(\rho)=\top \vee f_{2}(\rho)=\top
\end{aligned}
$$

This gives the standard set-theoretic semantics of first-order classical logic over $\mathcal{A}$, namely $\mathcal{I}_{\mathcal{A}} \llbracket-\rrbracket: S_{\Pi_{\Sigma \text {-fol }}} \rightarrow H^{I_{\mathcal{A}}}$.

Predicates: Subscone We introduce the notion of predicates over a categorical interpretation of types. When types are interpreted in set theory, the natural notion of predicate is simply a subset of each carrier set. In categorical settings, carrier sets are replaced by objects, and the notion of predicates is more subtle.

We write $\operatorname{Sub}(\mathbb{D})$ for the category of subobjects in a category $\mathbb{D}$, and $p_{\mathbb{D}}: \operatorname{Sub}(\mathbb{D}) \rightarrow$ $\mathbb{D}$ for the forgetful functor. First we recall injective scones in [MS93]. The injective scone of a Cartesian category $\mathbb{C}$ is the category obtained by pulling back $p_{\text {Set }}$ along the global section functor $\mathbb{C}(1,-)$ ([Jac99], example 1.5.2). In this approach, the notion of predicates over an object $C$ in $\mathbb{C}$ is represented as subsets of global elements of $C$.

In this paper we use the subscone approach [Laf88,MR92,MS93,PPST00], which is a mild generalisation of injective scones. We replace Set with a category $\mathbb{D}$ with finite limits and the global section functor with finite-product preserving functor. We define the category $\operatorname{Pred}(G)$ of $G$-predicates by pulling back $p_{\mathbb{D}}$ along $G$.

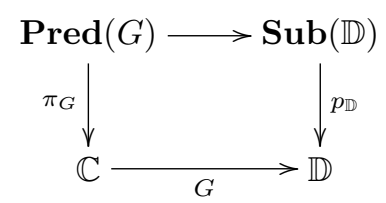

Category $\operatorname{Pred}(G)$ has finite products which are strictly preserved by $\pi_{G}{ }^{4}$. We also define the category $\operatorname{Rel}_{2}(G)$ of binary $G$-relations to be $\operatorname{Pred}(\operatorname{prod} \circ G \times G$ ), where prod : $\mathbb{C} \times \mathbb{C} \rightarrow \mathbb{C}$ gives the binary products in $\mathbb{C}$.

We adopt the following notational convention. Let $P$ and $Q$ be objects in $\operatorname{Pred}(G)$ and $f: \pi_{G}(P) \rightarrow \pi_{G}(Q)$ be a morphism in $\mathbb{C}$. We write $f: P \rightarrow Q$ if there exists a morphism $g: P \rightarrow Q$ in $\operatorname{Pred}(G)$ such that $\pi_{G}(g)=f$.

Let $F: T \rightarrow \mathbb{C}$ and $P: T \rightarrow \operatorname{Pred}(G)$ be interpretations of types. We say that $P$ is a $G$-predicate over $F$ (written $P \subseteq_{G} F$ ) if $\pi_{G} \circ P=F$. A binary $G$-relation $P$ between $F_{1}, F_{2}: T \rightarrow \mathbb{C}$ (written $P \subseteq_{G} F_{1}, F_{2}$ ) is just a (prodo $G \times G$ )-predicate over $\left\langle F_{1}, F_{2}\right\rangle$. For a predicate $P \subseteq_{G} F$, there exists a monomorphism $H^{\pi_{G}}: H^{P} \longmapsto H^{F}$.

\footnotetext{
${ }^{4}$ We give a proof in terms of fibred category theory. In fact $p_{\mathbb{D}}$ is a fibration with fibred finite limits, thus so is $\pi_{G}$ (see [Jac99], section 1.8). Then it follows from lemma 8.5.2 of [Jac99].
} 


\section{Pre-logical Predicates}

In this section, we fix a Cartesian category $\mathbb{C}$, a category $\mathbb{D}$ with finite limits, a finite product preserving functor $G: \mathbb{C} \rightarrow \mathbb{D}$ and a binding signature $\Pi$.

Let $\mathbb{C} \llbracket-\rrbracket_{F}$ be a categorical interpretation of $\Pi$ and $P \subseteq_{G} F$ be a predicate. We consider taking pullback of $H^{\pi_{G}}$ along $\mathbb{C} \llbracket-\rrbracket_{F}$ in $\mathbf{M}_{T}$.

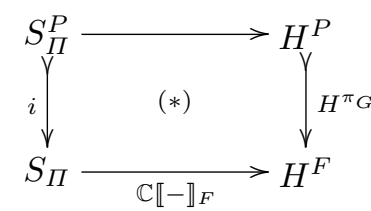

The vertex $S_{\Pi}^{P}$ can be calculated as $S_{\Pi}^{P}(\tau)(\Gamma)=\left\{M \mid \Gamma \vdash_{\Pi} M: \tau \wedge \mathbb{C} \llbracket M \rrbracket_{F}\right.$ : $\left.P^{*} \Gamma \rightarrow P \tau\right\}$. This represents the collection of terms whose meanings by $\mathbb{C} \llbracket-\rrbracket_{F}$ respects the predicate $P$. Thus when this is isomorphic to $S_{\Pi}$, the meanings of all the well-formed terms by $\mathbb{C} \llbracket-\rrbracket_{F}$ respects the predicate $P$.

Definition 1. Let $\mathbb{C} \llbracket-\rrbracket_{F}$ be a categorical interpretation of $\Pi$. We say that a predicate $P \subseteq_{G} F$ satisfies the basic lemma for $\Pi$ along $\mathbb{C} \llbracket-\rrbracket_{F}$ if in diagram $(*), S_{\Pi}^{P}$ is isomorphic to $S_{\Pi}$. This is equivalent to say that there exists a necessarily unique morphism $p: S_{\Pi} \rightarrow H^{P}$ (convention: we use the small letter of the predicate) such that $H^{\pi_{G}} \circ p=\mathbb{C} \llbracket-\rrbracket_{F}$.

Example 3. (Continued from example 2)

1. Let $P \subseteq \mathbb{C}(1,-) F_{\lambda}$ be a predicate satisfying the basic lemma for $\Pi_{\lambda}$ along $\mathbb{C} \llbracket-\rrbracket_{F_{\lambda}}$. It is equivalent to the $\mathbf{T y p} \vec{p}(B)$-indexed family of subsets $P \tau \subseteq \mathbb{C}\left(1, F_{\lambda} \tau\right)$ such that for all $\rho \in P^{*} \Gamma$ and $\Gamma \vdash_{\Pi_{\lambda}} M: \tau$, we have $\mathbb{C} \llbracket M \rrbracket_{F_{\lambda}} \circ \rho \in P \tau$.

2. Let $P \subseteq \operatorname{Id}_{\text {Set }} A$ be a predicate satisfying the basic lemma for $\Sigma$ along $\mathcal{A} \llbracket-\rrbracket$. It is equivalent to the $T_{0}$-indexed family of subsets $P \tau \subseteq A \tau$ satisfying $\mathcal{A} \llbracket M \rrbracket \rho \in P \tau$ for all $\Gamma \vdash_{\Sigma} M: \tau$ and $\rho \in P^{*} \Gamma$.

3. Let $\Sigma$ be a first-order signature, $\mathcal{A}, \mathcal{B}$ be many-sorted $\Sigma$-algebras and $P \subseteq{ }_{\text {Id }}$ set $I_{\mathcal{A}}, I_{\mathcal{B}}$ be a binary relation satisfying the basic lemma for $\Pi_{\Sigma \text {-fol }}$ along $\mathcal{I}_{\mathcal{A}} \llbracket-\rrbracket \times$ $\mathcal{I}_{\mathcal{B}} \llbracket-\rrbracket$ and $P \boldsymbol{\Omega}=\mathrm{id}_{\boldsymbol{\Omega}}$. It is equivalent to a $T_{0} \cup\{\boldsymbol{\Omega}\}$-indexed family of subsets $P \tau \subseteq A \tau \times B \tau$ such that for all $\Gamma \vdash_{\Pi_{\Sigma-\text { fol }}} M: \tau$ and $\left(\rho, \rho^{\prime}\right) \in P^{*} \Gamma$, $\left(\mathcal{I}_{\mathcal{A}} \llbracket M \rrbracket \rho, \mathcal{I}_{\mathcal{B}} \llbracket M \rrbracket \rho^{\prime}\right) \in P \tau$ when $\tau \in T_{0}$ and $\mathcal{I}_{\mathcal{A}} \llbracket M \rrbracket \rho=\mathcal{I}_{\mathcal{B}} \llbracket M \rrbracket \rho$ when $\tau=\boldsymbol{\Omega}$. The latter implies that $\mathcal{A}$ and $\mathcal{B}$ are elementary equivalent.

Now we introduce the notion of pre-logical predicates.

Definition 2. Let $\mathbb{C} \llbracket-\rrbracket_{F}$ be a categorical interpretation of $\Pi$. We call a predicate $P \subseteq_{G} F$ pre-logical for $\Pi$ along $\mathbb{C} \|-\rrbracket_{F}$ if in diagram $(*)$ there exists a necessarily unique $\Pi$-algebra $\left(S_{\Pi}^{P}, \alpha: \Pi S_{\Pi}^{P} \rightarrow S_{\Pi}^{P}\right)$ such that the projection $i$ induced by pullback is a $\Pi$-algebra morphism to the initial algebra $\left(S_{\Pi}, \iota_{\Pi}\right)$.

An elementary description of $P$ being pre-logical is that a) for all $\Gamma \vdash_{\Pi} x^{\tau}: \tau$, $\mathbb{C} \llbracket x^{\tau} \rrbracket_{F}: P^{*} \Gamma \rightarrow P \tau$ and $\left.\mathrm{b}\right)$ for all operator $o \in O$ of arity $\left(\overrightarrow{\tau_{1}}, \sigma_{1}\right), \cdots,\left(\overrightarrow{\tau_{m}}, \sigma_{m}\right) \rightarrow$ 
$\tau$ and well-formed terms $\Gamma, \overrightarrow{x_{i}: \tau_{i}} \vdash_{\Pi} M_{i}: \sigma_{i}(1 \leq i \leq n), \mathbb{C} \llbracket M_{i} \rrbracket_{F}: P^{*}\left(\Gamma, \overrightarrow{x_{i}: \tau_{i}}\right) \rightarrow$ $P \sigma_{i}$ for all $1 \leq i \leq n$ implies $\mathbb{C} \llbracket o\left(\overrightarrow{x_{1}^{\tau_{1}}} \cdot M_{1}, \cdots, \overrightarrow{x_{n}^{\tau_{n}}} \cdot M_{n}\right) \rrbracket_{F}: P^{*} \Gamma \rightarrow P \tau$. Normally a) is satisfied as variables are interpreted by projections. For operators having no variable binding, if the interpretation of terms $\mathbb{C} \llbracket-\rrbracket_{F}$ satisfies a) and the semantic substitution lemma, i.e. $\mathbb{C} \llbracket M\left[\overrightarrow{M_{i} / x_{i}}\right] \rrbracket_{F}=\mathbb{C} \llbracket M \rrbracket_{F} \circ\left\langle\overrightarrow{\mathbb{C} \llbracket M_{i} \rrbracket_{F}}\right\rangle$, then the condition b) can be rewritten to $\mathbb{C} \llbracket s^{\tau_{1}, \cdots, \tau_{n} \rightarrow \tau}\left(x_{1}^{\tau_{1}}, \cdots, x_{n}^{\tau_{n}}\right) \rrbracket_{F} \in P^{*}(\overrightarrow{x: \tau}) \rightarrow P \tau$.

Example 4. (Continued from example 2)

1. A predicate $P \subseteq \mathbb{C}(1,-), F_{\lambda}$ is pre-logical for $\Pi_{\lambda}$ along $\mathbb{C} \llbracket-\rrbracket_{F_{\lambda}}$ if for all $f \in$ $P\left(\tau \Rightarrow \tau^{\prime}\right)$ and $g \in P \tau$, @ $\circ\langle f, g\rangle \in P \tau^{\prime}$, and for all $\Gamma, x: \tau \vdash_{\Pi_{\lambda}} M: \tau^{\prime}$, $\forall \rho \in P^{*}(\Gamma, x: \tau) . \mathbb{C} \llbracket M \rrbracket_{F_{\lambda}} \circ \rho \in P \tau^{\prime}$ implies $\forall \rho \in P^{*} \Gamma . \mathbb{C} \llbracket \lambda x: \tau . M \rrbracket_{F_{\lambda}} \circ \rho \in$ $P\left(\tau \Rightarrow \tau^{\prime}\right)$.

2. A predicate $P \subseteq \operatorname{Id}_{\text {Set }} A$ is pre-logical for $\Sigma$ along $\mathcal{A} \llbracket-\rrbracket$ if for all $c^{\tau_{1}, \cdots, \tau_{n} \rightarrow \tau} \in O_{0}$ and $x_{i} \in P^{\tau_{i}}, c_{\mathcal{A}}^{\tau_{1}, \cdots, \tau_{n} \rightarrow \tau}\left(x_{1}, \cdots, x_{n}\right) \in P^{\tau}$. An algebraic predicate [HS02] is just a pre-logical predicate for $\Sigma_{C L}$ along $\mathcal{U} \llbracket-\rrbracket$ for a typed combinatory algebra $\mathcal{U}$ (i.e. a many-sorted $\Sigma_{C L}$ algebra).

3. A predicate $P \subseteq \operatorname{Id}_{\text {Set }} I_{\mathcal{A}}$ is pre-logical for $\Pi_{\Sigma \text {-fol }}$ along $\mathcal{I}_{\mathcal{A}} \llbracket-\rrbracket$ if for all first-order operator (including or, not) $s^{\tau_{1}, \cdots, \tau_{n} \rightarrow \tau}$ and $x_{i} \in P^{\tau_{i}}, s_{\mathcal{A}}^{\tau_{1}, \cdots, \tau_{n} \rightarrow \tau}\left(x_{1}, \cdots, x_{n}\right) \in$ $P^{\tau}$ holds, and for all $\tau \in T_{0}$ and $\Gamma, x: \tau \vdash M: \Omega, \forall \rho \in P^{*}(\Gamma, x: \tau) . \mathcal{I}_{\mathcal{A}} \llbracket M \rrbracket \rho \in$ $P \boldsymbol{\Omega}$ implies $\forall \rho \in P^{*} \Gamma . \mathcal{I}_{\mathcal{A}} \llbracket \operatorname{exists}(x . M) \rrbracket \rho \in P \boldsymbol{\Omega}$.

Theorem 1 (The Basic Lemma of Pre-logical Predicates). Let $\mathbb{C} \llbracket-\rrbracket_{F}$ be a categorical interpretation of $\Pi$. A predicate $P \subseteq_{G} F$ is pre-logical if and only if $P$ satisfies the basic lemma.

Proof. (if) If $P$ satisfies the basic lemma, we have an isomorphism $f: S_{\Pi}^{P} \cong S_{\Pi}$. Then $f:\left(S_{\Pi}^{P}, f^{-1} \circ \iota_{\Pi} \circ(\Pi f)\right) \rightarrow\left(S_{\Pi}, \iota_{\Pi}\right)$ is a $\Pi$-algebra morphism. Therefore $P$ is pre-logical.

(only if) Suppose there exists a $\Pi$-algebra $\left(S_{\Pi}^{P}, \alpha\right)$. Let ! $\left(S_{\Pi}, \iota_{\Pi}\right) \rightarrow\left(S_{\Pi}^{P}, \alpha\right)$ be the unique morphism from the initial $\Pi$-algebra. From the universal property of initial $\Pi$-algebra, we have $i \circ !=i d$. Now we have $i \circ ! \circ i=i=i \circ i d$, and since $i$ is mono, $! \circ i=i d$. Therefore $\left(S_{\Pi}^{P}, \alpha\right)$ and $\left(S_{\Pi}, \iota_{\Pi}\right)$ are isomorphic, thus $S_{\Pi}$ and $S_{\Pi}^{P}$ are so.

This theorem is a categorical re-formulation of the inductive proof of the basic lemma for pre-logical relations in [HS02]. From now on we identify pre-logical predicates and predicates satisfying the basic lemma.

We give one sufficient condition for $P$ being pre-logical. Below we identify a monomorphism in $\mathbf{M}_{T}$ and an object in $\operatorname{Sub}\left(\mathbf{M}_{T}\right)$. First, we can lift the endofunctor (of a typed binding signature) $\Pi$ to the one over $\operatorname{Sub}\left(\mathbf{M}_{T}\right)$, namely $\tilde{\Pi}$. Here lifting means that $\tilde{\Pi}$ satisfies $p_{\mathbf{M}_{T}} \circ \tilde{\Pi}=\Pi \circ p_{\mathbf{M}_{T}}$ (see [Jac99], section 9.2). This is because all the constructs of $\Pi$ have liftings over $\mathbf{S u b}\left(\mathbf{S}_{T}\right)$. Functor $p_{\mathbf{M}_{T}}$ is a subobject fibration, thus admits comprehension ([Jac99], example 4.6.3). It is easy to see that $\tilde{\Pi} \circ \top \cong \top \circ \Pi$, where $\top: \mathbf{M}_{T} \rightarrow \operatorname{Sub}\left(\mathbf{M}_{T}\right)$ is the right adjoint of $p_{\mathbf{M}_{T}}$ giving fibred terminal objects. Thus an initial $\Pi$-algebra is inductive ([Jac99], definition 9.2.6, proposition 9.2.7), i.e. $i d_{S_{\Pi}}$ is an initial $\tilde{\Pi}$-algebra. 
Proposition 1. Let $P \subseteq G$ F be a predicate and suppose that $H^{\pi_{G}}: H^{P} \longmapsto H^{F}$ has a $\tilde{\Pi}$-algebra structure. Then $P$ satisfies the basic lemma for $\Pi$ along the initial algebra semantics of $\Pi$ in $H^{F}$.

\section{Composability of Pre-logical Relations}

We move to the composability of binary pre-logical relations. Binary pre-logical relations are closed under relational composition, which is not enjoyed by logical relations [HS02]. We give here a categorical account of composability of pre-logical relations. In this section we fix a typed binding signature $\Pi$, a Cartesian category $\mathbb{C}$, a category $\mathbb{D}$ with finite limits, a finite-product preserving functor $G: \mathbb{C} \rightarrow \mathbb{D}$ and categorical interpretations $\mathbb{C} \llbracket-\rrbracket_{F_{i}}(1 \leq i \leq 3)$ of $\Pi$. We write fst, snd : $\mathbb{C} \times \mathbb{C} \rightarrow \mathbb{C}$ for projections.

First, we assume that a composition operator $c$ over $\operatorname{Rel}_{2}(G)$ is available. This operator is partial, and defined over composable pairs of relations, i.e. a pair $(R, S)$ of objects in $\operatorname{Rel}_{2}(G)$ such that $\operatorname{snd}\left(\pi_{G}(R)\right)=\operatorname{fst}\left(\pi_{G}(S)\right)$. The composition operator yields an object $c(R, S)$ in $\operatorname{Rel}_{2}(G)$ such that $\operatorname{fst}\left(\pi_{G}(c(R, S))\right)=\operatorname{fst}\left(\pi_{G}(R)\right)$ and $\operatorname{snd}\left(\pi_{G}(c(R, S))\right)=\operatorname{snd}\left(\pi_{G}(S)\right)$, and a morphism $c(f, g): c(R, S) \rightarrow c\left(R^{\prime}, S^{\prime}\right)$ for composable pairs of relations $(R, S),\left(R^{\prime}, S^{\prime}\right)$ and morphisms $f: R \rightarrow R^{\prime}, g$ : $S \rightarrow S^{\prime}$ in $\operatorname{Rel}_{2}(G)$. It is natural to assume that $\operatorname{Rel}_{2}(G)$ has identity relation, and the composition operator satisfies the laws of identity and associativity. To summarise, we assume that we have a category object in Cat:

$$
\operatorname{Rel}_{c}(G) \stackrel{c}{\longrightarrow} \operatorname{Rel}_{2}(G) \underset{\partial_{2}=\mathrm{sndo} \pi_{G}}{\stackrel{\partial_{1}=\mathrm{fsto} \pi_{G}}{\rightleftarrows}} \mathbb{C}
$$

where $\operatorname{Rel}_{c}(G)$ is the category of composable pairs of relations obtained by pulling back $\partial_{2}$ along $\partial_{1}$. Using category objects in Cat to formulate the composition of relations is due to [KOPT97].

For $R \subseteq_{G} F_{1}, F_{2}$ and $S \subseteq G F_{2}, F_{3}$, we define their composition $c(R, S)(\tau)$ to be $c(R \tau, S \tau)$. It is clear that $c(R, S) \subseteq G F_{1}, F_{3}$.

Theorem 2 (Composability of Pre-logical Relations). Let $R \subseteq_{G} F_{1}, F_{2}$ and $S \subseteq G$ $F_{2}, F_{3}$ be pre-logical binary relations for $\Pi$ along $\mathbb{C} \llbracket-\rrbracket_{F_{1}} \times \mathbb{C} \llbracket-\rrbracket_{F_{2}}$ and $\mathbb{C} \llbracket-\rrbracket_{F_{2}} \times$ $\mathbb{C} \llbracket-\rrbracket_{F_{3}}$ respectively. Then $c(R, S) \subseteq_{G} F_{1}, F_{3}$ is pre-logical for $\Pi$ along $\mathbb{C} \llbracket-\rrbracket F_{1} \times$ $\mathbb{C} \llbracket-\rrbracket_{F_{3}}$.

Proof. We find a morphism $h: S_{\Pi} \rightarrow H^{c(R, S)}$ such that $H^{\pi_{G}} \circ h=\mathbb{C} \llbracket-\rrbracket_{F_{1}} \times$ $\mathbb{C} \llbracket-\rrbracket_{F_{3}}$ where $H^{\pi_{G}}: H^{c(R, S)} \rightarrow H^{\left\langle F_{1}, F_{3}\right\rangle}$ is the morphism in $\mathbf{M}_{T}$. We give $h$ by $h(\tau)(\Gamma)(M)=c(r(\tau)(\Gamma)(M), s(\tau)(\Gamma)(M))$ for all well-formed terms $\Gamma \vdash_{\Pi} M: \tau$, where $r: S_{\Pi} \rightarrow H^{R}$ and $s: S_{\Pi} \rightarrow H^{S}$ are morphisms which exist by definition of the basic lemma (see definition 1).

When do we have a category object as $(* *)$ above? Recall that composition of relations can be expressed by the $c(R, S)(x, z)=\exists y \cdot R(x, y) \wedge S(y, z)$. The standard interpretation of this formula in set theory gives the composition of binary relations. Now we replace set theory with regular fibration [Jac99], which is a preordered fibration $p: \mathbb{E} \rightarrow \mathbb{B}$ such that $\mathbb{B}$ is Cartesian and $p$ has fibred finite products, fibred 
equality and simple coproducts satisfying Frobenius and Beck-Chevalley (for details, see [Jac99]). A regular fibration provides a categorical model of the $\exists \wedge \top=$-fragment of predicate logic. Interpreting the above formula in this model gives rise to a composition operation, which enjoys the identity and associativity laws.

Proposition 2. Assume that $p_{\mathbb{D}}: \operatorname{Sub}(\mathbb{D}) \rightarrow \mathbb{D}$ is a regular fibration. Then we can construct a category object as (**) above in Cat.

\section{Examples}

Example 5. In this example, we examine the relationship between pre-logical predicates for combinatory algebras and pre-logical predicates for the simply typed lambda calculus in our framework. This is a revisit of proposition 3.3 in [HS02].

The standard abstraction mechanism $\lambda^{*} x . M$ in combinatory logic (see definition 7.1.5, [Bar84]) induces a $\Pi_{\lambda}$-algebra structures over $S_{\Sigma_{C L}}$. From the universal property of initial $\Pi_{\lambda}$-algebra, there is a unique $\Pi_{\lambda}$-algebra morphism, namely $(-)_{C L}$ : $S_{\Pi_{\lambda}} \rightarrow S_{\Sigma_{C L}}$, which coincides with the standard lambda-to-CL translation (definition 7.3.1, [Bar84]). The composition $\mathcal{U} \llbracket(-)_{C L} \rrbracket$ gives an interpretation of the simply typed lambda calculus in a combinatory algebra $\mathcal{U}$. In general this is not a $\Pi_{\lambda}$-algebra morphism. Conversely, giving the standard representation of $S, K$ combinators in $S_{\Pi_{\lambda}}$ equips it with a $\Sigma_{C L}$ algebra structure. Then there exists a unique $\Sigma_{C L}$-algebra morphism from an initial $\Sigma_{C L}$-algebra, namely $(-)_{\lambda}: S_{\Sigma_{C L}} \rightarrow S_{\Pi_{\lambda}}$.

Let $\mathcal{U}$ be a combinatory algebra and $P \subseteq \operatorname{Id}_{\text {Set }} U$ be a pre-logical predicate for $\Sigma_{C L}$ along $\mathcal{U} \llbracket-\rrbracket$. Then we have $H^{\pi_{G}} \circ p \circ(-)_{C L}=\mathcal{U} \llbracket(-)_{C L} \rrbracket$, thus $P$ is pre-logical for $\Pi_{\lambda}$ along $\mathcal{U} \llbracket(-)_{C L} \rrbracket$. This explains that an algebraic predicate relating combinators yields a pre-logical predicate ("if" part of proposition 3.3, [HS02]). Conversely, let $P \subseteq \operatorname{Id}_{\text {Set }} U$ be a pre-logical predicate for $\Pi_{\lambda}$ along $\mathcal{U} \llbracket(-)_{C L} \rrbracket$. It is a pre-logical predicate for $\Sigma_{C L}$ along $\mathcal{U} \llbracket\left((-)_{\lambda}\right)_{C L} \rrbracket-$ but not for $\mathcal{U} \llbracket-\rrbracket$ in general!

Theorem 3. There exists a combinatory algebra $\mathcal{U}_{0}$ and a pre-logical predicate $P \subseteq \operatorname{Id}_{\text {Set }}$ $U_{0}$ for $\Pi_{\lambda}$ along $\mathcal{U}_{0} \llbracket(-)_{C L} \rrbracket$ which is not not pre-logical for $\Pi_{C L}$ along $\mathcal{U}_{0} \llbracket-\rrbracket$.

The proof uses the fact that the image of the standard lambda-to-CL translation does not cover the entire set of combinatory logic terms, particularly $S$ and $K$. To exploit this fact, we take $\mathcal{U}_{0}$ as the closed term algebra, and see that the definability predicate by $\mathcal{U}_{0} \llbracket(-)_{C L} \rrbracket$, which is pre-logical for $\Pi_{\lambda}$, is not pre-logical for $\Pi_{C L}$ along $\mathcal{U}_{0} \llbracket-\rrbracket$. This means that "only if" part of proposition 3.3, [HSO2] is not precise enough. The subtle point is that "to which semantics" it satisfies the basic lemma, and it was missed in [HS02].

When is $P$ a pre-logical predicate for $\Sigma_{C L}$ along $\mathcal{U} \llbracket-\rrbracket$ ? One answer is to fix the lambda-to-CL translation $(-)_{C L}$ to make it surjective. To achieve this, we introduce another abstraction mechanism $\lambda^{\prime} x . M$ defined to be $\lambda^{\prime} x . x=S K K, \lambda^{\prime} x . M=K M$ provided $x \notin \mathrm{FV}(M), \lambda^{\prime} x \cdot \lambda^{*} y \cdot x=K, \lambda^{\prime} x \cdot \lambda^{*} y \cdot \lambda^{*} z \cdot x z(y z)=S, \lambda^{\prime} x \cdot M N=$ $S\left(\lambda^{\prime} x . M\right)\left(\lambda^{\prime} x . N\right)$. The lambda-to-CL translation constructed from this abstraction mechanism, say $(-)_{C L^{\prime}}$, covers all the combinators, and moreover satisfies $\left((M)_{\lambda}\right)_{C L^{\prime}}=$ $M$. Thus a pre-logical predicate for $\Pi_{\lambda}$ along $\mathcal{U} \llbracket(-)_{C L^{\prime}} \rrbracket$ is a pre-logical predicate for 
$\Sigma_{C L}$ along $\mathcal{U} \llbracket-\rrbracket$. Another answer is to require $\mathcal{U}$ to be a lambda algebra, which always satisfies $\mathcal{U} \llbracket\left((-)_{\lambda}\right)_{C L} \rrbracket=\mathcal{U} \llbracket-\rrbracket$ (see lemma 5.2.3-2, [Bar84]).

Example 6. We examine the connection between lax logical predicates [PPST00] and pre-logical predicates as defined in here. For this, we fix a set of base types $B$ and define the set of types including finite products by the BNF Typ $\Rightarrow \times(B) \ni \tau::=$ $b|1| \tau \times \tau \mid \tau \Rightarrow \tau$ where $b \in B$. The signature for the simply typed lambda calculus with finite products is defined by

$$
\begin{aligned}
\Pi_{\lambda \times}= & \left(\mathbf{T y p}^{\Rightarrow} \Rightarrow \times(B),\left\{\operatorname{lam}^{\left(\tau, \tau^{\prime}\right) \rightarrow \tau \Rightarrow \tau^{\prime}}, \operatorname{app}^{\tau \Rightarrow \tau^{\prime}, \tau \rightarrow \tau^{\prime}}, *^{1},\right.\right. \\
& \text { pair } \left.\left.^{\tau, \tau^{\prime} \rightarrow \tau \times \tau^{\prime}}, \text { fst }^{\tau \times \tau^{\prime} \rightarrow \tau}, \text { snd }^{\tau \times \tau^{\prime} \rightarrow \tau^{\prime}}\right\}\right) .
\end{aligned}
$$

Let $\mathbf{L}$ be the free CCC generated from the set of base types $B$. An object of $\mathbf{L}$ is a type $\tau \in \mathbf{T y p}{ }^{\Rightarrow \times}(B)$, and a morphism from $\tau$ to $\tau^{\prime}$ in $\mathbf{L}$ is a $\beta \eta$-equivalence class of a wellformed terms $x: \tau \vdash_{\Pi_{\lambda \times}} M: \tau^{\prime}$. We write $I: \mathbf{T y p}^{\Rightarrow}{ }^{\times}(B) \rightarrow \mathbf{L}$ for the inclusion functor. As we have seen in example 2 , since $\mathbf{L}$ is a CCC, it provides a $\Pi_{\lambda \times}$-algebra structure, thus there exists a unique $\Pi_{\lambda \times}$-morphism $\mathbf{L} \llbracket-\rrbracket_{I}: S_{\Pi_{\lambda \times}} \rightarrow H^{I}$. We note that the mapping $\mathbf{L} \llbracket-\rrbracket_{I}$ is an epimorphism.

Let $\mathbb{C}$ be a CCC, $\mathbb{D}$ be a CCC with finite limits, $G: \mathbb{C} \rightarrow \mathbb{D}$ be a functor preserving finite products and $\llbracket-\rrbracket: \mathbf{L} \rightarrow \mathbb{C}$ be a strict Cartesian closed functor. A lax logical predicate $q[$ PPST00] over $\llbracket-\rrbracket$ is a finite-product preserving functor $q: \mathbf{L} \rightarrow \operatorname{Pred}(G)$ such that $\pi_{G} \circ q=\llbracket-\rrbracket$.

Theorem 4. A lax logical predicate $p: \mathbf{L} \rightarrow \operatorname{Pred}(G)$ determines a pre-logical predicate $p \circ I \subseteq_{G} \llbracket-\rrbracket \circ I$ for $\Pi_{\lambda \times}$ along $H^{\llbracket-\rrbracket} \circ \mathbf{L} \llbracket-\rrbracket_{I}$. Conversely, if $P \subseteq_{G} \llbracket-\rrbracket \circ I$ is a pre-logical predicate for $\Pi_{\lambda \times}$ along $H \llbracket-\rrbracket \circ \mathbf{L} \llbracket-\rrbracket_{I}$, then there exists a lax logical predicate $q$ such that for all $\Gamma \vdash_{\Pi_{\lambda \times}} M: \tau, H^{q} \circ \mathbf{L} \llbracket M \rrbracket_{I}=p(\tau)(\Gamma)(M)$.

Example 7. In this example we see a characterisation of elementary submodels in terms of a binary pre-logical relation. Let $\Sigma=\left(T_{0}, O_{0}\right)$ be a typed first-order signature, $\mathcal{B}$ be a many-sorted $\Sigma$-algebra and $\mathcal{A}$ be a subalgebra of $\mathcal{B}$. For all $\Gamma \vdash_{\Pi_{\Sigma \text {-fol }}} M: \tau$ with $\tau \in T_{0}$, we have $\mathcal{A} \llbracket M \rrbracket=\mathcal{B} \llbracket M \rrbracket$ because $\mathcal{A}$ is a submodel of $\mathcal{B}$. However, this may not hold when $\tau=\boldsymbol{\Omega}$ because of existential quantifier. Thus we say $\mathcal{A}$ is an elementary submodel of $\mathcal{B}$ (written $\mathcal{A} \preceq \mathcal{B}$ ) if the above holds for $\tau=\Omega$ as well.

Theorem 5. $\mathcal{A} \preceq \mathcal{B}$ if and only if the inclusion relation $R \subseteq_{\operatorname{Id}_{\text {Set }}} I_{\mathcal{A}}, I_{\mathcal{B}}$, i.e. $R^{\Omega}=$ $i d_{\Omega}$ and $R^{\tau}=\left\{(x, x) \mid x \in A^{\tau}\right\}$, is pre-logical for $\Pi_{\Sigma \text {-fol }}$ along $\mathcal{I}_{\mathcal{A}} \llbracket-\rrbracket \times \mathcal{I}_{\mathcal{B}} \llbracket-\rrbracket$.

\section{Conclusion}

We have given a generalisation of pre-logical predicates to arbitrary typed formal systems, and shown that they are equivalent to predicates satisfying the basic lemma, and that binary pre-logical relations are closed under composition. We represent three underlying components of pre-logical predicates - syntax, semantics and predicates in the category of presentation models. Then we formulate pre-logical predicates and predicates satisfying the basic lemma, and show their equivalence. 
It is interesting to extend our framework for defining formal systems. One direction is to allow type variables so that we can cover type systems such as System F or FPC [FP94]. The other direction is to modify the notion of contexts from the Cartesian one to linear one to cover linear logic. In both cases we also have to switch the notion of models from Cartesian categories to more elaborate categorical structures such as polymorphic fibrations, symmetric monoidal categories, etc.

Acknowledgments I thank Donald Sannella, Daniel Turi and John Power for useful discussions. This work was supported by an LFCS studentship.

\section{References}

[Bar84] H. Barendregt. The Lambda Calculus-Its Sytax and Semantics. North Holland, 1984.

[Fio02] M. Fiore. Semantic analysis of normalisation by evaluation for typed lambda calculus. In Proc. PPDP 2002, pages 26-37. ACM, 2002.

[FP94] M. Fiore and G. Plotkin. An axiomatization of computationally adequate domain theoretic models of FPC. In Proc. LICS 1994, pages 92-102. IEEE, 1994.

[FPT99] M. Fiore, G. Plotkin, and D. Turi. Abstract syntax and variable binding. In Proc. LICS 1999, pages 193-202. IEEE Computer Society Press, 1999.

[Her93] C. Hermida. Fibrations, Logical Predicates and Indeterminantes. PhD thesis, The University of Edinburgh, 1993.

[HLST00] F. Honsell, J. Longley, D. Sannella, and A. Tarlecki. Constructive data refinement in typed lambda calculus. In Proc. FoSSACS 2000, volume 1784 of LNCS, pages 161-176. Springer, 2000.

[Hof99] M. Hoffman. Semantical analysis of higher-order abstract syntax. In Proc. LICS 1999, pages 204-213. IEEE Computer Society, 1999.

[HS02] F. Honsell and D. Sannella. Prelogical relations. INFCTRL: Information and Computation (formerly Information and Control), 178(1):23-43, 2002.

[Jac99] B. Jacobs. Categorical Logic and Type Theory. Elsevier, 1999.

[Kat03] S. Katsumata. Behavioural equivalence and indistinguishability in higher-order typed languages. In WADT 2002, Revised Selected Papers, volume 2755 of LNCS, pages 284-298. Springer, 2003.

[KOPT97] Y. Kinoshita, P. W. O’Hearn, A. J. Power, and M. Takeyama. An axiomatic approach to binary logical relations with applications to data refinement. In Proc. TACS 1997, volume 1281 of $L N C S$, pages 191-212. Springer, 1997.

[KP99] Y. Kinoshita and J. Power. Data-refinement for call-by-value programming languages. In Proc. CSL 1999, volume 1683 of LNCS, pages 562-576. Springr, 1999.

[Laf88] Y. Lafont. Logiques, Categories et Machines. PhD thesis, Université de Paris VII, 1988.

[Lei01] H. Leiß. Second-order pre-logical relations and representation independence. In Proc. TLCA 2001, volume 2044 of LNCS, pages 298-314. Springer, 2001.

[Mit96] J. Mitchell. Foundations for Programming Languages. MIT Press, 1996.

[MR92] Q. Ma and J. C. Reynolds. Types, abstractions, and parametric polymorphism, part 2. In In Proc. MFPS 1991, volume 598 of LNCS, pages 1-40. Springer, 1992.

[MS93] J. Mitchell and A. Scedrov. Notes on sconing and relators. In Proc. CSL 1992, volume 702 of $L N C S$, pages 352-378. Springer, 1993.

[MS03] M. Miculan and I. Scagnetto. A framework for typed HOAS and semantics. In Proc. PPDP 2003, pages 184-194. ACM, 2003.

[PPST00] G. Plotkin, J. Power, D. Sannella, and R. Tennent. Lax logical relations. In Proc. ICALP 2000, volume 1853 of LNCS, pages 85-102. Springer, 2000. 


\section{A Proofs}

Proof of proposition 1 The initiality of $i d_{S_{\Pi}}$ gives a morphism $f: S_{\Pi} \rightarrow H^{P}$ which is above the $\Pi$-algebra morphism ! : $S_{\Pi} \rightarrow H^{F}$, i.e. $H^{\pi_{G}} \circ f=$ !. Thus $P$ satisfies the basic lemma by definition.

Proof of proposition 2 To prove this proposition, we use the internal logic of fibrations [Jac99]. From the assumption, the logic provides the $\exists \wedge \top=$-fragment of predicate logic. For details, see [Jac99]. The following reasoning is done in the internal logic of $p_{\mathbb{D}}$.

An object in $\operatorname{Rel}_{2}(G)$ is a triple $\left(C, C^{\prime}, P\right)$ where $C, C^{\prime}$ are objects in $\mathbb{C}$ and $P$ is a predicate $x: G C, y: G C^{\prime} \vdash P(x, y)$ of the internal logic of the fibration. A morphism from $\left(C, C^{\prime}, P\right)$ to $\left(D, D^{\prime}, Q\right)$ is a pair of morphisms $f: C \rightarrow D, g: C^{\prime} \rightarrow D^{\prime}$ in $\mathbb{C}$ such that $x: G C, y: G C^{\prime} \mid P(x, y) \vdash Q(G f(x), G g(y))$ holds.

An object in $\operatorname{Rel}_{c}(G)$ is a tuple $\left(C, C^{\prime}, C^{\prime \prime}, P, P^{\prime}\right)$ such that $\left(C, C^{\prime}, P\right)$ and $\left(C^{\prime}, C^{\prime \prime}, P^{\prime}\right)$ are objects in $\operatorname{Rel}_{2}(G)$. A morphism from $\left(C, C^{\prime}, C^{\prime \prime}, P, P^{\prime}\right)$ to $\left(D, D^{\prime}, D^{\prime \prime}, Q, Q^{\prime}\right)$ in $\operatorname{Rel}_{c}(G)$ is a triple $\left(f: C \rightarrow D, g: C^{\prime} \rightarrow D^{\prime}, h: C^{\prime \prime} \rightarrow D^{\prime \prime}\right)$ such that $(f, g):\left(C, C^{\prime}, P\right) \rightarrow\left(D, D^{\prime}, Q\right)$ and $(g, h):\left(C^{\prime}, C^{\prime \prime}, P^{\prime}\right) \rightarrow\left(D^{\prime}, D^{\prime \prime}, Q^{\prime}\right)$ are morphisms in $\mathbf{R e l}_{2}(G)$.

For an object $C$ in $\mathbb{C}$, we assign an object $i d(C)$ in $\operatorname{Rel}_{2}(G)$ by $x: G C, y: G C \vdash$ $x=y$. For all $f: C \rightarrow D$ in $\mathbb{C}$, we can derive a judgment $x: G C, y: G C \vdash x=y \vdash$ $G f(x)=G f(y)$ in the internal logic of the fibration. We can extend this assignment to a functor $\mathbb{C} \rightarrow \operatorname{Rel}_{2}(G)$.

For an object $\left(C, C^{\prime}, C^{\prime \prime}, P, P^{\prime}\right)$ in $\operatorname{Rel}_{c}(G)$, we define an object $c\left(C, C^{\prime}, C^{\prime \prime}, P, P^{\prime}\right)$ in $\operatorname{Rel}_{2}(G)$ by $C, C^{\prime \prime}, x: G C, z: G C^{\prime \prime} \vdash \exists y: G C^{\prime} . P(x, y) \wedge P^{\prime}(y, z)$ (we omit reindexing functors along projections for readability).

Let $(f, g, h):\left(C, C^{\prime}, C^{\prime \prime}, P, P^{\prime}\right) \rightarrow\left(D, D^{\prime}, D^{\prime \prime}, Q, Q^{\prime}\right)$ be a morphism in $\operatorname{Rel}_{c}(G)$. In the internal logic of the fibration, we have the following derivation (annotation of objects are omitted for readability):

$\frac{\frac{x, y \mid P(x, y) \vdash Q(G f(x), G g(y))}{x, y, z \mid P(x, y) \wedge P^{\prime}(y, z) \vdash Q(G f(x), G g(y))} \frac{y, z \mid P^{\prime}(y, z) \vdash Q^{\prime}(G g(y), G h(z))}{x, y, z \mid P(x, y) \wedge P^{\prime}(y, z) \vdash Q^{\prime}(G g(y), G h(z))}}{\frac{x, y, z \mid P(x, y) \wedge P^{\prime}(y, z) \vdash Q(G f(x), G g(y)) \wedge Q^{\prime}(G g(y), G h(z))}{\frac{x, y, z \mid P(x, y) \wedge P^{\prime}(y, z) \vdash \exists y \cdot Q(G f(x), y) \wedge Q^{\prime}(y, G h(z))}{x, z \mid \exists y \cdot P(x, y) \wedge P^{\prime}(y, z) \vdash \exists y \cdot Q(G f(x), y) \wedge Q^{\prime}(y, G g(z))}}}$

Thus $c$ extends to a functor $c: \operatorname{Rel}_{c}(G) \rightarrow \operatorname{Rel}_{2}(G)$.

To see that $i d$ and $c$ satisfy the laws of category object, such as $c\left(i d(C),\left(C, C^{\prime}, P\right)\right)=$ $\left(C, C^{\prime}, P\right)$, we show that the predicates on both sides are provably equal. The calculation is much like that in [Jac99], example 4.3.8. Since $p_{\mathbb{D}}$ is a fibred partial order, provable equality implies equality of objects. Thus the above equation strictly holds.

Proof of theorem 3 We use the fact that the image of the standard lambda-to-CL translation does not cover the entire set of combinatory logic terms. 
First we write $[M]_{w}$ for the equivalence class of a combinatory logic term $M$ by weak equivalence $={ }_{w}$ (see [Bar84], definition 7.2.1). We define the closed term combinatory algebra $\mathcal{U}_{0}$ by the tuple $\left(U_{0}, \bullet_{w},[S]_{w},[K]_{w}\right)$ where $U_{0}^{\tau}=\left\{[M]_{w} \mid M \in\right.$ $\left.S_{\Sigma_{C L}}(\emptyset, \tau)\right\}$ and $\bullet_{w}^{\tau, \tau^{\prime}}$ is the application operators defined by $[M]_{w} \bullet \bullet_{w}^{\tau, \tau^{\prime}}[N]_{w}=$ $[M N]_{w}$ for $[M]_{w} \in U_{0}^{\tau \Rightarrow \tau^{\prime}}$ and $[N]_{w} \in U_{0}^{\tau}$. It is easy to see that the above choice of combinators satisfies the axioms of the combinatory algebra. As we have seen in example 2 , we obtain an interpretation of combinatory logic terms in $\mathcal{U}_{0}$, namely $\mathcal{U}_{0} \llbracket-\rrbracket$. Explicitly, $\mathcal{U}_{0} \llbracket M \rrbracket\left\{\left[M_{1}\right]_{w} / x_{1}, \cdots,\left[M_{n}\right]_{w} / x_{n}\right\}=\left[M\left[M_{1} / x_{1}, \cdots, M_{n} / x_{n}\right]\right]_{w}$. We interpret simply typed lambda terms by $\mathcal{U}_{0} \llbracket(-)_{C L} \rrbracket$.

Now we define the definability predicate $D^{\tau} \subseteq U_{0}^{\tau}$ by $D^{\tau}=\left\{\mathcal{U}_{0} \llbracket M_{C L} \rrbracket \in\right.$ $\left.U_{0}^{\tau} \mid M \in S_{\Pi_{\lambda}}(\emptyset, \tau)\right\}$. This is a pre-logical predicate for $\Pi_{\lambda}$ along $\mathcal{U}_{0} \llbracket(-)_{C L} \rrbracket$. However, $D^{\tau \Rightarrow \tau^{\prime} \Rightarrow \tau}$ does not include $\left[K^{\tau \Rightarrow \tau^{\prime} \Rightarrow \tau}\right]_{w}$ for all $\tau$ and $\tau^{\prime}$, thus is not a pre-logical predicate for $\Sigma_{C L}$ along $\mathcal{U}_{0} \llbracket-\rrbracket$.

It is easy to see that there exists no closed term $M$ such that $M_{C L}=K$ by induction on $M$. Next we prove the following lemma:

Lemma 1. For all closed lambda term $M$ and all combinatory term $N, M_{C L} \rightarrow w N$ implies there exists a closed lambda term $N^{\prime}$ such that $N=N_{C L}^{\prime}$.

When $M$ begins with a lambda abstraction, $M_{C L}$ is always a normal form. Thus the claim clearly holds by taking $N^{\prime}=M$. We do not consider the case when $M$ is a variable, since we assume $M$ is closed. So we think of the case when $M=M_{0} M_{1}$ with two closed lambda terms $M_{0}$ and $M_{1}$. There are several possible causes of $M_{C L} \rightarrow w$ $N$.

- $N=M_{C L}$. We just take $N^{\prime}=M$.

- There exists a combinatory term $L$ such that $\left(M_{0}\right)_{C L} \rightarrow_{w} L$ and $L \rightarrow_{w} N$. From $\mathrm{IH}$, there exists a combinatory term $L^{\prime}$ such that $L=L_{C L}^{\prime}$. Again from $\mathrm{IH}$, there exists a combinatory term $N^{\prime}$ such that $N=N_{C L}^{\prime}$.

- $\left(M_{0}\right)_{C L} \rightarrow w N_{0}$ and $N=N_{0}\left(M_{1}\right)_{C L}$. From IH, there exists a closed lambda term $N_{0}^{\prime}$ such that $\left(N_{0}^{\prime}\right)_{C L}=N_{0}$. Thus $N=\left(N_{0}^{\prime}\right)_{C L}\left(M_{1}\right)_{C L}=\left(N_{0}^{\prime} M_{1}\right)_{C L}$.

- $\left(M_{1}\right)_{C L} \rightarrow{ }_{w} N_{1}$ and $N=\left(M_{0}\right)_{C L} N_{1}$. The proof is similar to the above case.

- $\left(M_{0}\right)_{C L}=K N_{0}$ with a combinatory term $N_{0}$ and $N=N_{0}$. From the definition of lambda-to-CL translation, $M_{0}$ should be equal to $\lambda x . N_{0}^{\prime}$ where $N_{0}^{\prime}$ is a closed lambda term. Thus $N_{0}=\left(N_{0}^{\prime}\right)_{C L}$.

- $\left(M_{0}\right)_{C L}=S N_{0} N_{1}$ with some combinatory terms $N_{0}, N_{1}$ and $N=N_{0}\left(M_{1}\right)_{C L}\left(N_{1}\left(M_{1}\right)_{C L}\right)$. From the definition of lambda-to-CL translation, $M_{0}$ should be equal to $\lambda x$. $\left(N_{0}^{\prime} N_{1}^{\prime}\right)$. Then $\lambda x \cdot\left(N_{0}^{\prime} N_{1}^{\prime}\right)=S\left(N_{0}^{\prime}\right)_{C L}\left(N_{1}^{\prime}\right)_{C L}$, which implies $N_{0}=\left(N_{0}^{\prime}\right)_{C L}$ and $N_{1}=$ $\left(M_{1}^{\prime}\right)_{C L}$. Thus we take $N^{\prime}=N_{0}^{\prime} M_{1}\left(N_{1}^{\prime} M_{1}\right)$.

Thus there exists no term reducing to $K$ in the image of $(-)_{C L}$, otherwise $K$ should be in the image of $(-)_{C L}$ (we assume the strong noramlisation of $\rightarrow w$ ). Thus $[K]_{w} \notin$ $D^{\tau \Rightarrow \tau^{\prime} \Rightarrow \tau}$.

Proof of theorem 4 We only show the converse. The assumption says that there exists a morphism $p: S_{\Pi_{\lambda \times}} \rightarrow H^{P}$ such that $H^{\pi_{G}} \circ p=H^{\llbracket-\rrbracket} \circ \mathbf{L} \llbracket-\rrbracket_{I}$. Recall that $\mathbf{L} \llbracket-\rrbracket_{I}$ is an epimorphism and $H^{\pi_{G}}$ is a monomorphism. In category $\mathbf{M}_{T}$, any epimorphism is 
orthogonal to monomorphism, thus there exists a unique morphism $h$ such that $H^{\pi_{G}}$ 。 $h=H^{\llbracket-\rrbracket}$ and $h \circ \mathbf{L} \llbracket-\rrbracket_{I}=p$. Now we define the functor $q: \mathbf{L} \rightarrow \operatorname{Pred}(G)$ in question by $q \tau=P \tau$ and $q f=h\left(\tau^{\prime}\right)(\tau)(f)$ for a morphism $f: \tau \rightarrow \tau^{\prime}$ in $\mathbf{L}$. We see $q$ is indeed a functor. First $q$ preserves identity, since $\pi_{G}\left(q\left(i d_{\tau}\right)\right)=\llbracket i d_{\tau} \rrbracket=i d_{\llbracket \tau \rrbracket}=\pi_{G}\left(i d_{P \tau}\right)$ and $\pi_{G}$ is faithful, we have $q\left(i d_{\tau}\right)=i d_{P \tau}$. Next we show $q(f \circ g)=q f \circ q g$ for all $f: \tau^{\prime} \rightarrow \tau^{\prime \prime}$ and $g: \tau \rightarrow \tau^{\prime}$. We have $\pi_{G}(q(f \circ g))=\llbracket f \circ g \rrbracket=\llbracket f \rrbracket \circ \llbracket g \rrbracket=$ $\pi_{G}(q f \circ q g)$, and since $\pi_{G}$ is faithful, we have $q(f \circ g)=q f \circ q g$. It is routine to check $H^{q} \circ \mathbf{L} \llbracket M \rrbracket_{I}=p(\tau)(\Gamma)(M)$.

Next we show $P\left(\tau \times \tau^{\prime}\right)=P \tau \times P \tau^{\prime}$. We consider well-formed terms $x: \tau \times$ $\tau^{\prime} \vdash_{\Pi_{\lambda \times}}$ fst $(x): \tau$ and $x: \tau \times \tau^{\prime} \vdash_{\Pi_{\lambda \times}}$ snd $(x): \tau$. We define $j=\langle p(\tau)(x:$ $\left.\left.\tau \times \tau^{\prime}\right)(\mathrm{fst}(x)), p\left(\tau^{\prime}\right)\left(x: \tau \times \tau^{\prime}\right)(\operatorname{snd}(x))\right\rangle$. Since $H^{\pi_{G}} \circ p=\llbracket-\rrbracket \circ \mathbf{L} \llbracket-\rrbracket_{I}$, we can show that $\pi_{G} j=\mathrm{id}_{\llbracket \tau \times \tau^{\prime} \rrbracket}$, which implies $j=\mathrm{id}_{P\left(\tau \times \tau^{\prime}\right)}$ since $\pi_{G}$ is faithful. This means that the comparison map $P\left(\tau \times \tau^{\prime}\right) \rightarrow P \tau \times P \tau^{\prime}$ is identity, thus $P\left(\tau \times \tau^{\prime}\right)=P \tau \times P \tau^{\prime}$ holds.

Proof of theorem 5 Assume $\mathcal{A} \preceq \mathcal{B}$. We only have to show that for all $\Gamma \vdash_{\Pi_{\Sigma \text {-fol }}} M: \tau$ where $\tau \in T_{0}$ and $\rho \in A^{*} \Gamma, \mathcal{I}_{\mathcal{A}} \llbracket M \rrbracket \rho=\mathcal{I}_{\mathcal{B}} \llbracket M \rrbracket \rho$. This is clear, since $M$ consists of operators in $\Sigma$, and $\mathcal{A}$ is a subalgebra of $\mathcal{B}$. Conversely, assume that the basic lemma holds. Then for all $\Gamma \vdash_{\Pi_{\Sigma \text {-fol }}} M: \Omega$ and $\rho \in A^{*} \Gamma$, we have $\left(\mathcal{I}_{\mathcal{A}} \llbracket M \rrbracket \rho, \mathcal{I}_{\mathcal{B}} \llbracket M \rrbracket \rho\right) \in$ $R \Omega=\operatorname{id}_{\Omega}$. Thus $\mathcal{A} \preceq \mathcal{B}$ holds. 